\title{
Fentanilo intranasal para el tratamiento del dolor irruptivo oncológico
}

\author{
M.A. Vidal, J.M. Trinidad y L.M. Torres \\ Servicio de Anestesia y Tratamiento del Dolor. Hospital Universitario Puerta del Mar. Cádiz
}

Vidal MA, Trinidad JM, Torres LM. Fentanilo intranasal para el tratamiento del dolor irruptivo oncológico. Rev Soc Esp Dolor 2014; 21(2): 106-111.

\begin{abstract}
Breakthrough oncological pain (BOP) consists of a transient exacerbation on a persistent otherwise basal controlled pain. It is a moderate or severe pain that reaches the peak of intensity quickly and has a relatively short duration. These features make fentanyl a good treatment option due its high lipophilicity and quick absorption. Here takes on special importance the intranasally route which, by its high vascularization and permeability, constitutes a particularly quick route. Recently, fentanyl mixed with a solution of pectin called PecFent ${ }^{\circledR}$ nasal spray has been developed. The objective of this study is to conduct a review of published studies on its use for the treatment of BOP. Randomized, controlled, double-blind trials have shown an onset of the pain relief in just 5 minutes after its administration, as well as in 10 min clinically meaningful pain relief. Despite being the current standard treatment of immediate release morphine sulfate, comparative studies have shown the superiority of intranasal fentanyl to treat this type of episodes. Intranasal fentanyl achieves a faster than other drugs and clinically meaningful pain relief. The administration by intranasal fentanyl has proven to be well tolerated. Clinical trials program is has found the presence of typical adverse effects of the drugs opioid in this population. The most common were vomiting, nausea, progression of the disease and constipation, the majority being mild to moderate in intensity. The nasal route did not damage by continued use of intranasal fentanyl.
\end{abstract}

Key words: Intranasal fentanyl. Breakthrough oncological pain. Cancer. Efficiency. Tolerance.

\section{RESUMEN}

El dolor irruptivo (DIO) consiste en una exacerbación transitoria sobre un dolor de base persistente, por lo demás controlado. Se trata de un dolor moderado o intenso, que alcanza el pico de intensidad rápidamente y tiene una duración relativamente corta. Estas características hacen del fentanilo una buena opción de tratamiento por su alta lipofilia y capacidad de absorción rápida. Aquí cobra especial importancia la vía intranasal que, por su alta vascularización y permeabilidad, constituye una ruta especialmente rápida. Recientemente se ha desarrollado un espray nasal de fentanilo mezclado con una solución de pectina denominado PecFent ${ }^{\circledR}$. El objetivo de este estudio es realizar una revisión de los estudios publicados sobre su uso para el tratamiento del DIO. Ensayos aleatorizados, controlados, a doble ciego han demostrado un inicio del alivio del dolor en tan sólo 5 minutos tras su administración, así como el alivio del dolor clínicamente significativo en $10 \mathrm{~min}$. A pesar de ser el sulfato morfina de liberación inmediata el tratamiento estándar actual de los episodios de DIO, estudios comparativos han demostrado la superioridad del fentanilo intranasal para tratar este tipo de episodios porque logra un alivio del dolor clínicamente significativo y más rápido que otros fármacos. La administración por vía nasal del fentanilo ha demostrado ser bien tolerada. En el programa de ensayos clínicos se ha objetivado la presencia de efectos adversos típicos de los medicamentos opioides en esta población. Los más frecuentes fueron vómitos, náuseas, progresión de la enfermedad y estreñimiento, siendo la mayoría de leves a moderados en intensidad. La vía nasal no presentó daños por el uso continuado de fentanilo intranasal.

Palabras clave: Fentanilo intranasal. Dolor irruptivo. Cáncer. Eficacia. Tolerancia. 


\section{INTRODUCCIÓN}

El fentanilo intranasal está indicado para el tratamiento del dolor irruptivo en los adultos que estén recibiendo tratamiento de mantenimiento con opioides para el dolor oncológico crónico.

El dolor irruptivo consiste en una exacerbación transitoria sobre un dolor de base persistente, por lo demás controlado (1). Se sabe que lo padecen el 33-95\% de los pacientes con cáncer $(2,3)$.

El dolor irruptivo oncológico (DIO) se caracteriza por ser un dolor moderado o intenso que suele ser imprevisible, alcanza el pico de intensidad de forma muy rápida (1-3 minutos) y de relativamente corta duración (una media de 45 minutos) $(2,4)$.

Históricamente este tipo de dolor se ha tratado con opioides de liberación rápida por vía oral, siendo el sulfato de morfina de liberación inmediata el tratamiento estándar actual (5). Sin embargo, por sus características farmacocinéticas la morfina por esta vía de administración puede no proporcionar un inicio de acción óptimo para el DIO y además provocar efectos secundarios cuando el dolor desparece y la morfina continúa actuando $(6,7)$.

Es por esto que se han buscado alternativas con fármacos con mejor perfil farmacocinético que se adecuen más a las características del DIO, como es el caso del fentanilo, dada su alta lipofilia y su capacidad de absorción rápida a través de mucosas $(8,9)$. Se han llevado a cabo estudios con distintas vías de administración: transmucosas (bucal y sublingual) e intranasal. Se han objetivado como principal limitación de las fórmulas para su absorción a través de la mucosa bucal los problemas bucales que suelen presentar los pacientes oncológicos, como la xerostomía, presente en más de $78 \%$ de los casos $(10,11)$. Por esto cobra especial importancia la administración intranasal, que por la alta vascularización y permeabilidad de la mucosa nasal constituye una ruta especialmente rápida e indicada para el DIO $(12,13)$.

Recientemente se ha desarrollado un espray nasal de fentanilo mezclado con una solución en pectina denominado PecFent ${ }^{\mathbb{R}}$. Utiliza la tecnología intranasal denominada PecSys ${ }^{\circledR}$, que permite formar en la cavidad nasal un gel de pectina que facilita la rápida absorción y alta biodisponibilidad del fentanilo (14).

El objetivo de este estudio es realizar una revisión de los estudios publicados sobre el uso del fentanilo intranasal para el tratamiento del DIO.

\section{MATERIAL}

Se buscaron sistemáticamente informes de ensayos controlados aleatorios (ECA) de fentanilo en el tratamiento del dolor usando las siguientes bases de datos: PubMed, Emba- se, Google Scholar y Cochrane, sin restricción de idioma. Se utilizaron combinaciones de texto libre incluyendo los siguientes términos de búsqueda: dolor oncológico, cáncer, nasal, aerosol, prueba de dolor, dolor y fentanilo. La última búsqueda se realizó el 3 de enero de 2012.

\section{RESULTADOS}

La autorización de comercialización de este fármaco se basa en una amplia revisión de datos clínicos que incluyen tres estudios en fase III con más de 650 pacientes y más de 100 centros de investigación y 13 países. De los diversos estudios realizados para valorar la eficacia y tolerabilidad del PecFent ${ }^{\circledR}$ destacamos los siguientes:

- Estudio de fase III, doble ciego, aleatorizado, controlado con placebo, cruzado y multicéntrico en el que participaron clínicas de dolor, servicios de anestesia, unidades de cuidados paliativos y clínicas oncológicas de Austria, Dinamarca, Francia, Alemania y Polonia. Se incluyeron pacientes oncológicos que tomaban un tratamiento de mantenimiento con opioides y sufrían un mínimo de 3 episodios semanales de DIO y un máximo de 4 al día, con una duración mayor a $15 \mathrm{~min}$.

Durante una fase inicial fueron randomizados para recibir tratamiento domiciliario con dosis de 50, 100 o $200 \mu \mathrm{g}$ de espray de fentanilo intranasal frente a placebo durante 3 semanas para controlar el DIO. En esta fase de ajuste de dosis se daba por válida si controlaba 3 o 4 episodios de DIO (con una reducción de la intensidad del dolor de 2 o más puntos tras la administración de la medicación, sin la presencia de efectos adversos). La respuesta fue medida por una escala categórica del 0 al $4(0=$ pobre, $1=$ escasa, $2=$ buena, $3=$ muy buena $y$ $4=$ excelente). La variable principal del estudio fue la diferencia en la intensidad del dolor a los 10 min tras la administración del fármaco, utilizando una escala numérica (del 0 al 10) a los 10, 20, 40 y 60 minutos tras la administración del fármaco. Se valoró la comparación entre la diferencia de las sumas de la intensidad del dolor a los 60 minutos de la administración del medicamento y la impresión general de eficacia en el mismo tiempo. Un total de 111 pacientes recibieron tratamiento con espray nasal de fentanilo, frente a 110 que recibieron placebo. La diferencia en la intensidad del dolor a los 10 minutos tras la dosis de fentanilo intranasal fue de 2,36 vs. 1,10 con placebo. Las 3 dosis resultaron clínicamente eficaces y bien toleradas. Hubo una diferencia significativa en la reducción del dolor de los episodios de DIO con el tratamiento con fentanilo intranasal frente a placebo $(\mathrm{p}<0,001)$. La 
prevalencia de efectos adversos fue de un 19,8 \%, siendo los más frecuentes náuseas $(4,5 \%)$ y vértigo $(1,8 \%)$. En total, 108 pacientes participaron en la fase abierta de tolerabilidad del estudio, con una media de exposición al tratamiento de 134,9 días, durante los cuales el efecto adverso más común fue la progresión de la enfermedad (50,9\%) (15).

- Estudio multicéntrico, doble ciego, aleatorizado, controlado con placebo y cruzado en el que se incluyeron 114 pacientes que tomaban un tratamiento de mantenimiento con opioides y sufrían un promedio de $1 \mathrm{a}$ 4 episodios de dolor irruptivo al día (estudio CP043). Constaba de una fase inicial y abierta de ajuste de dosis de fentanilo intranasal, seguido de una fase de doble ciego en la que los pacientes recibieron aleatoriamente fentanilo intranasal o placebo para el tratamiento de 7 o 3 episodios de DIO, respectivamente. Se valoró la intensidad del dolor (escala del 0 al 10) y el alivio del mismo (escala del 0 al 5) a los 5, 10, $15,30,45$ y 60 minutos del tratamiento, así como la diferencia en la intensidad del dolor con respecto al basal y la suma de las diferencias de la intensidad de dolor. El criterio principal de valoración fue la comparación entre la diferencia de la intensidad del dolor a los 30 minutos de la administración del medicamento $\left(\operatorname{DSID}_{30}\right)$. También se recogió la utilización de medicación de rescate y la aceptabilidad por parte del paciente, medida en una escala categórica del 0 al 3, a los 30 y 60 minutos de la administración de la dosis. De los pacientes que entraron en la fase de ajuste de dosis, en un $6 \%$ fue insuficiente y un $5 \%$ abandonó el estudio por los efectos adversos. La $\operatorname{DSID}_{30}$ fue de 6,57 en los episodios tratados con fentanilo, en comparación con 4,45 en el caso del placebo $(\mathrm{p}<$ $0,00001)$. Las puntuaciones medias de la intensidad del dolor de todos los episodios tratados con fentanilo (459 episodios), en comparación con los tratados con placebo (200 episodios), fueron significativamente inferiores a los $5,10,15,30,45$ y 60 minutos de la administración. Un $33 \%$ de los 459 episodios de DIO tratados con fentanilo intranasal obtuvo una reducción clínicamente significativa del dolor frente al placebo a los $10 \min (p=0,011)$, aumentando a un $66 \%$ a los $30 \min (\mathrm{p}<0,0001 v s$. placebo), obteniendo una reducción de la intensidad del dolor $\geq 2$ puntos tras la administración del fármaco $(\mathrm{p}<0,01)$. La necesidad de recurrir a medicación de rescate fue menor en el grupo tratado con fentanilo frente al placebo $(p<0,001)$. La aceptación por parte del paciente fue mayor en términos de comodidad y satisfacción. Los efectos adversos fueron más frecuentes en el grupo tratado con fentanilo, presentando vómitos $(10,6 \%)$, náuseas $(8,8 \%)$, mareos $(8 \%)$, epistaxis $(4,4 \%)$ y nasofaringitis $(3,5 \%)(16)$.
- Estudio de diseño similar al anterior, multicéntrico (llevado a cabo en 36 centros de Estados Unidos, Costa Rica y Argentina), doble ciego, aleatorizado, controlado con placebo y cruzado, en el cual se incluyeron 114 pacientes que tomaban un tratamiento de mantenimiento con opioides y sufrían un promedio de 1 a 4 episodios de dolor irruptivo al día. Constaba de una fase inicial y abierta de ajuste de dosis de fentanilo intranasal, seguido de una fase de doble ciego en la que los pacientes recibieron aleatoriamente fentanilo intranasal o placebo para el tratamiento de 7 o 3 episodios de DIO respectivamente. Un total de 83 pacientes entraron en la segunda fase del estudio. Comparado con placebo, el tratamiento con fentanilo intranasal consiguió una mayor diferencia de la intensidad del dolor desde los 10 minutos de la administración del medicamento $(\mathrm{p}<0,05)$ hasta los 60 minutos ( $p<0,0001)$, incluida la variable principal del estudio a los 30 minutos $\left(\operatorname{DSID}_{30}\right)(\mathrm{p}<0,0001)$. Aproximadamente el $70 \%$ de los pacientes se mostró satisfecho o muy satisfecho con el tratamiento con fentanilo intranasal, resultando seguro y bien tolerado. Únicamente un 5,3\% de los pacientes dejó el tratamiento debido a los efectos adversos, sin identificarse efectos nasales significativos (17).

Al considerarse el sulfato de morfina el tratamiento estándar de los episodios de DIO, se han llevado a cabo estudios comparativos con este fármaco:

- Estudio multicéntrico, doble ciego, aleatorizado, controlado con sulfato de morfina y cruzado en el que se incluyeron 110 pacientes que tomaban un tratamiento de mantenimiento con opioides y sufrían un promedio de 1 a 4 episodios de dolor irruptivo al día. Un total de 84 pacientes completaron la primera fase de tratamiento y entraron en la segunda fase del estudio, en la que de forma aleatoria recibieron fentanilo intranasal o cápsulas por vía oral de placebo para el tratamiento de 5 episodios de DIO o sulfato de morfina de liberación inmediata o espray nasal de placebo para el tratamiento de otros 5 episodios de DIO. La variable principal del estudio fue la diferencia de la intensidad del dolor a los 15 minutos de recibir el tratamiento, comprobándose en el estudio la eficacia y tolerabilidad del fentanilo intranasal y la capacidad de conseguir analgesia de forma más rápida que con el sulfato de morfina de liberación inmediata (18).

- Estudio multicéntrico, doble ciego, aleatorizado, controlado con sulfato de morfina y cruzado de diseño similar al anterior. Se valoró la intensidad del dolor y el alivio de este y la aceptación por parte de los pacientes a los 30 y 60 minutos. La seguridad y tolerabilidad se midió por los efectos adversos y las reacciones locales. En el estudio se obtuvo un alivio del dolor de forma más rápida con el fentanilo intranasal que con 
la morfina de liberación inmediata; a los 10 minutos se obtuvieron diferencias significativas en la intensidad del dolor y en el porcentaje de episodios, que consiguió un alivio del dolor clínicamente significativo $(\mathrm{p}<0,05)$. La aceptación por parte del paciente fue mayor en el grupo tratado con fentanilo a los $30(\mathrm{p}<0,01)$ y 60 minutos $(\mathrm{p}<0,05)$, estando satisfechos/muy satisfechos con la comodidad $(79,8 \%)$ y facilidad de su uso $(77,2 \%)$ en el grupo tratado con fentanilo. Un 7,4\% abandonó el estudio debido a los efectos adversos, sin objetivarse reacciones locales (19).

Dadas las características del DIO, se ha comparado el espray nasal de fentanilo con el fentanilo oral transmucosa, cuyo perfil farmacocinético parece más adecuado para el tratamiento del DIO:

- Estudio multicéntrico, randomizado, abierto y cruzado. El estudio constaba de una primera fase de ajuste de dosis en la que un total de 139 pacientes fueron asignados de forma aleatoria para recibir tratamiento con 50, 100 o $200 \mu \mathrm{g}$ de fentanilo intranasal seguido de 200, 400, 600, 800, 1.200 o $1.600 \mu \mathrm{g}$ de fentanilo oral transmucosa, o viceversa, para el tratamiento de 6 episodios de DIO. En una segunda fase los pacientes recibían la dosis titulada de cada fármaco y se comparaban en términos de intensidad de dolor, alivio del dolor y facilidad de uso. La variable principal del estudio fue el tiempo en el que el paciente tardó en percibir un alivio significativo del dolor. Otras variables del estudio fueron la diferencia de la intensidad del dolor a los 10 y 30 minutos -PID (10), PID (30)-, la suma de la diferencia de la intensidad del dolor a los 15 y 60 minutos -SPID (15), SPID (60)-, la facilidad de administración y la preferencia de tratamiento. Los investigadores calcularon la relación existente entre las dosis requeridas para el tratamiento de los episodios de DIO y las dosis analgésicas programadas. Finalmente se calculó la proporción de episodios con una reducción de la intensidad del dolor $\geq 33 \%$ $\mathrm{y} \geq 50 \%$ a los $5,10,15,20,30$ y 60 minutos. De los 139 pacientes randomizados para recibir ambos tratamientos 53 no completaron esta fase, siendo la proporción de abandonos similar con ambos tratamientos. Se trataron 577 episodios de DIO con cada uno de los tratamientos. El $75 \%$ de los pacientes tratados con fentanilo intranasal necesitó dosis de 100-200 $\mu \mathrm{g}$ y el $60 \%$ de los tratados con fentanilo oral transmucosa dosis de 200-400 $\mu \mathrm{g}$. El tiempo medio para conseguir un alivio significativo del dolor fue de 11 minutos tras el tratamiento con fentanilo intranasal frente a 16 minutos en caso de fentanilo oral transmucosa. Un $65,7 \%$ de los pacientes experimentó un inicio más rápido del alivio del dolor con el fentanilo intranasal $(\mathrm{p}<0,001)$. La PID fue mayor de forma estadísticamente significativa desde los 5 minutos tras la admi- nistración del fentanilo intranasal. El grupo tratado con fentanilo intranasal consiguió una proporción mayor de reducción del $33 \%$ y del $50 \%$ de la intensidad del dolor. El $56 \%$ de los pacientes experimentó al menos un efecto adverso, siendo la mayoría de carácter moderado. Los pacientes prefirieron el tratamiento con fentanilo intranasal $(\mathrm{p}<0,001)$ y lo consideraron más fácil de utilizar. Ambos tratamientos fueron bien tolerados. El único efecto adverso que se dio con una frecuencia $>5 \%$ en ambos tratamientos fueron las náuseas (20).

Se han realizado estudios a largo plazo con el fin de comprobar la seguridad del fentanilo intranasal:

- Estudio de seguridad, multicéntrico, abierto y a largo plazo en el que se incluyeron 403 pacientes con dolor crónico oncológico, controlado con tratamiento opiáceo y que sufrían 1-4 episodios de DIO al día (estudio 045). Tras una fase de ajuste de dosis, 356 pacientes entraron en la fase de tratamiento de 16 semanas, durante la cual 42.227 episodios de DIO recibieron tratamiento con fentanilo intranasal. Se valoró la seguridad y tolerabilidad, recogiendo los efectos adversos sistémicos y locales. También se recogió la necesidad de administrar medicación de rescate, así como la necesidad de aumentar la dosis de fentanilo intranasal. Durante la fase de tratamiento un 24,6\% de los pacientes relató efectos adversos moderados o graves relacionados con el tratamiento opiáceo. De los 80 fallecimientos ocurridos durante el estudio 1 pudo considerarse como posible consecuencia del tratamiento. No se objetivaron efectos adversos nasales. Tras la administración de fentanilo intranasal no se requirió medicación de rescate en un $94 \%$ de los casos. Y en más de un $90 \%$ de los pacientes no hubo que aumentar la dosis inicial de fentanilo intranasal $(21,22)$.

\section{DISCUSIÓN}

Las características clínicas del DIO, hacen que el tratamiento ideal sea un medicamento potente con un inicio de acción en cuestión de minutos y una duración analgésica de menos de 1 hora, constituyendo fentanilo en aplicación intranasal la mejor alternativa disponible actualmente.

PecFent ${ }^{\circledR}$ consiste en un espray intranasal de fentanilo en pectina que proporciona un alivio del dolor clínicamente significativo y más rápido que los fármacos actualmente disponibles. Es el primer espray intranasal de fentanilo en pectina comercializado para proporcionar un alivio rápido del dolor ajustándose al perfil típico del DIO. Se administra en una o ambas fosas nasales, utilizando soluciones de 0 , 5,1 y $2 \mathrm{mg} / \mathrm{ml}$, correspondiéndose con dosis de 50, 100 y $200 \mu \mathrm{g}$ de fentanilo (23). El PecFent ${ }^{\circledR}$ utiliza el sistema Pec Sys ${ }^{\circledR}$ de administración nasal de fármacos para modular la administración y la absorción del fentanilo. Permite pul- 
verizar el producto en la zona frontal de la cavidad nasal como una vaporización fina de microgotas que se gelifican al entrar en contacto con los iones de calcio presentes en la mucosa nasal. El fentanilo se difunde desde el gel y, al ser muy lipofílico, se absorbe muy rápidamente a través de la mucosa nasal. De esta forma se restringe el valor máximo en la concentración plasmática $\left(\mathrm{C}_{\max }\right)$, al tiempo que se alcanza pronto este valor máximo $\left(\mathrm{t}_{\max }\right)(24)$. Tiene una biodisponibilidad cercana al $70 \%$, alcanzando el $\mathrm{t}_{\max }$ en 5-16 minutos (25).

Ensayos aleatorizados, controlados, de doble ciego han demostrado un inicio del alivio del dolor tan sólo 5 minutos tras la administración de fentanilo intranasal, así como el alivio del dolor clínicamente significativo en 10 minutos. En el mayor estudio clínico realizado en el mundo sobre el DIO en cáncer, en el $94 \%$ de los episodios de dolor irruptivo tratados con la dosis inicialmente titulada de PecFent ${ }^{\circledR}$ los pacientes no requirieron medicación adicional de rescate.

A pesar de ser el sulfato morfina de liberación inmediata el tratamiento estándar actual de los episodios de DIO, estudios comparativos han demostrado la superioridad del fentanilo intranasal para tratar este tipo de episodios, ya que consigue un alivio del dolor clínicamente significativo de más intensidad y más rápidamente.

Buscando alternativas cuyo perfil farmacocinético se adecue más a las características del DIO, se ha comparado PecFent ${ }^{\mathbb{R}}$ con el fentanilo oral transmucosa, demostrándose un inicio del alivio del dolor más rápido y ser más fácil de utilizar con PecFent ${ }^{\circledR}$.

Se han realizado estudios a largo plazo con el fin de comprobar la seguridad del fentanilo intranasal. Cuando el fentanilo se administra por vía intranasal puede ocasionar efectos locales (como irritación nasal) y los efectos adversos sistémicos propios de los opioides (12). La administración por vía nasal del fentanilo ha demostrado ser bien tolerada. En el programa de ensayos clínicos, el uso de PecFent ${ }^{\mathbb{B}}$ en el tratamiento del DIO estaba asociado con la ocurrencia de efectos adversos típicos de los medicamentos opioides en esta población. Los más frecuentes fueron vómitos, náuseas, progresión de la enfermedad y estreñimiento, siendo la mayoría leves o moderados en intensidad.

PecFent ${ }^{\mathbb{R}}$ ofrece una nueva esperanza para pacientes que sufren de episodios de DIO atroces, debilitantes e inadecuadamente controlados, proporcionando un tratamiento muy rápido, eficaz, consistente y fácil de administrar, permitiendo una analgesia eficaz y segura controlada por el paciente.

\section{CORRESPONDENCIA:}

Luis Miguel Torres

Servicio de Anestesia y Tratamiento del Dolor

Hospital Universitario Puerta del Mar

Avda. Ana de Viya, 21.

11009 Cádiz

e-mail:1mtorres@arrakis.es

\section{BIBLIOGRAFÍA}

1. Mercadente S, Radbruch A, Caraceni N, Cherny N, Kaasa S, Nauck F, et al. De Conno, episodic (breakthrough) pain: Consensus conference o fan expert working group of the European Association for Palliative Care. Cancer 2002;94:832-9.

2. Portenoy R, Bruns D, Shoemaker B, Shoemaker S. Breakthrough pain in community-dwelling patients with cancer pain and noncancer pain 1: Prevalence and characteristics. J Opiod Manag 2010;6:97-108.

3. Svedsen KB, Andersen S, Arnason S, Arner S, Breivik H, Heiskanen T, et al. Breakthrough pain in malignant and non-malignant diseases: a review of prevalence, characteristics and mechanisms. Eur J Pain 2005;9:195-206.

4. Portenoy RK, Hagen NA. Breakthrough pain: Definition, prevalence and characteristics. Pain 1990;41:273-81.

5. Ruiz-Garcia V, Lopez-Briz E. Morphine remains gold standard in breakthrough cancer pain. BMJ 2008;337:3104.

6. Zeppetella G. Dynamics of breakthrough pain vs. Pharmacokinetics of oral morphine: Implications for management. Eur J Cancer Care (Engl) 2009; 18:331-7.

7. Zeppetella G. Opioids for cancer breakthrough pain: a pilot study reporting patient assessment of time to meaningful pain relief. J Pain Symptom manage 2008;35:563-7.

8. Darwish M, Kirby M, Robertson P, Hellriegel E and Jiang JG. Comparison of equivalent doses of fentanyl bucal tablets and arteriovenous differences in fentanyl pharmacokinetics. Clin Pharmacokinet 2006;45:843-50.

9. Vasisht N, Gever LN, Tagarro I, Finn AL. Formulation selection and pharmacokinetic comparison of fentanyl bucal soluble film wih oral transmucosal fentanyl citrate: a randomized, open-label, single-dose, crossover study. Clin drug Investig 2009;29:647-54.

10. Davies AN, Vriens J. Oral transmucosal fentanyl citrate and xerostomia. J Pain Symptom Manage 2005;30:496-7.

11. Jacobsen R, Moldrup C, Christrup L. Clinical rationale for administering fentanyl to cancer pain patients: Two Delphi surveys of pain management experts in Denmark. J Opioid Manage 2008;4:383-91

12. Dale O, Hjortkjaer R, Kharasch ED. Nasal administration of opioids for pain management in adults. Acta Anaesthesiol Scand 2002;46:759-70.

13. Shelley K, Paech MJ. The clinical applications of intranasal opioids. Curr Drug Deliv 2008;5:55.

14. Watts Pand Smith A. PecSys: In situ gelling system for optimised nasal drug delivery. Expert OPin Drug Deliv 2009;6:543-52.

15. Kress HG, Orońska A, Kaczmarek Z, Kaasa S, Colberg T, Nolte T. Efficacy and tolerability of intranasal fentanyl spray 50 to 200 microg for breakthrough pain in patients with cancer: A phase III, multinational, randomized, double-blind, placebo-controlled, crossover trial with a 10-month, open-label extension treatment period. Clin Ther 2009;31(6):1177-91.

16. Taylor D, Galán V, Wenstein SM, Reyes E, Pupo-Araya AR, ET AL. Fentanyl pectin nasal spray in breakthrogh pain. J Support Oncol 2010;8(4):184-90.

17. Portenoy RK, Burton AW, Gabrail N, Taylor D. A multicenter, placebo-controlled, double-blind, multiple-crossover study of Fentanyl Pectin Nasal Spray (FPNS) in the treatment of breakthrough cancer pain. Pain 2010;151(3):616-24.

18. Fallon M, Reale C, Davies A, Lux AE, Kumar K, Stachowiak A, et al. Efficacy and safety of fentanyl pectin nasal spray 
compared with inmediate-release morphine sulphate tablets in the treatment of breakthrough cancer pain: A multicenter, randomized, controlled, double-blind, double-dummy multiple-crossover study. J Support Oncol 2011;9(6):224-31.

19. Davies A, Sitte T, Elsner F, Reale C, Espinosa J, Brooks $\mathrm{D}$, et al. Consistence of efficacy, patient acceptability, and nasal spray compared with inmediate-release morphine sulfate in breakthrough cancer pain. J Pain Symptom Manage 2011;41(2):358-66.

20. Mercadante S, Radbruch L, Davies A, Poulain P, Sitte T, Perkins $P$, et al. A comparison of intranasal fentanyl spray with oral transmucosal fentanyl citrate for the treatment of breakthrough cancer pain: An open-label, randomised, crossover trial. Curr Med Res Opin 2009;25(11):2805-15.

21. Portenoy RK, Raffaeli W, Torres LM, Sitte T, Deka AC, Herrera IG, et al. Long-term safety, tolerability, and consistency of effect of fentanyl pectin nasal spray for breakthrough cancer pain in opioid-tolerant patients. J Opioid Manag 2010;6(5):319-28.
22. Radbruch L, Torres LM, Ellershaw JE, Gatti A, Luis Lerzo G, Revnic J, et al. Long-term tolerability, efficacy and acceptability of fentanyl pactin nasal spray for breakthrough cancer pain. Support Care Cancer 2012;20(3):565-73.

23. Panagiotou I, Mystakidou K. Intranasal fentanyl: From pharmacokinetics and bioavailability to current treatment applications. Expert Review of Anticancer Therapy 2010; 10(7):1009-1021.

24. Moksnes K, Fredheim OM, Klepstad P, Kaasa S, Angelsen A, Nielsen T, et al. Early pharmacokinetics of nasal fentayl: Is there a significant arterio-venous difference? Eur J Clin Pharmacol 2008;64:497-502.

25. Veldhorst-Janssen NM, Fiddelers AA, van der Kuy PH, Kessels AG, Theunissen HM, van der Hulst RR, et al. Pharmacokinetics, analgesic effect, and tolerability of a single preprocedural dose of intranasal fentanyl in patients undergoing drain removal after breast reduction or augmentation surgery: A prospective, randomized, double-blind, placebo-controlled study. Clin Ther 2010;32(7):1427-36. 